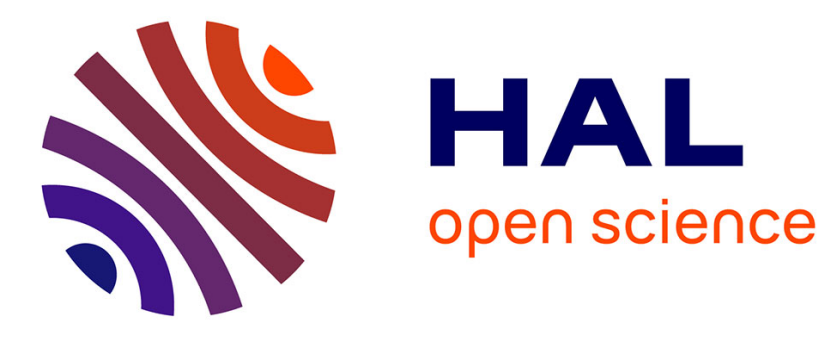

\title{
How mimetic should a robotic fish be to socially integrate into zebrafish groups?
}

Leo Cazenille, Bertrand Collignon, Yohann Chemtob, Frank Bonnet, Alexey

Gribovskiy, Francesco Mondada, Nicolas Bredeche, José Halloy

\section{- To cite this version:}

Leo Cazenille, Bertrand Collignon, Yohann Chemtob, Frank Bonnet, Alexey Gribovskiy, et al.. How mimetic should a robotic fish be to socially integrate into zebrafish groups?. Bioinspiration and Biomimetics, 2018, 13 (2), pp.025001. 10.1088/1748-3190/aa8f6a . hal-03313840

\section{HAL Id: hal-03313840 \\ https: / hal.sorbonne-universite.fr/hal-03313840}

Submitted on 5 Aug 2021

HAL is a multi-disciplinary open access archive for the deposit and dissemination of scientific research documents, whether they are published or not. The documents may come from teaching and research institutions in France or abroad, or from public or private research centers.
L'archive ouverte pluridisciplinaire HAL, est destinée au dépôt et à la diffusion de documents scientifiques de niveau recherche, publiés ou non, émanant des établissements d'enseignement et de recherche français ou étrangers, des laboratoires publics ou privés. 


\title{
How mimetic should a robotic fish be to socially integrate into zebrafish groups?
}

\author{
Leo Cazenille ${ }^{1,3}$, Bertrand Collignon ${ }^{1}$, Yohann Chemtob $^{1}$, Frank Bonnet ${ }^{2}$, Alexey Gribovskiy ${ }^{2}$, \\ Francesco Mondada ${ }^{2}$, Nicolas Bredeche ${ }^{3}$, José Halloy ${ }^{1}$ \\ 1 Univ Paris Diderot, Sorbonne Paris Cité, LIED, UMR 8236, 75013, Paris, France \\ 2 Robotic Systems Laboratory, School of Engineering, Ecole Polytechnique Fédérale de Lausanne, ME \\ B3 30, Station 9, 1015 Lausanne, Switzerland \\ 3 Sorbonne Universités, UPMC Univ Paris 06, CNRS, ISIR, F-75005 Paris, France
}

\begin{abstract}
Biomimetic robots are promising tools in animal behavioural studies. If they are socially integrated in a group of animals, they can produce calibrated social stimuli to test the animal responses. However, the design of such social robots is challenging as it involves both a luring capability including appropriate robot behaviours, and the acceptation of the robots by the animals as social companions. Here, we investigate the integration of a biomimetic robot driven by biomimetic behavioural models into a group of zebrafish (Danio rerio). The robot behaviours are based on a stochastic model linking zebrafish visual perception to individual behaviour and calibrated experimentally to correspond to the behaviour of zebrafish. We show that our robot can be integrated into a group of zebrafish, mimic their behaviour and exhibit similar collective dynamics compared to fish-only groups. This study shows that an autonomous biomimetic robot was enhanced by a biomimetic behavioural model so that it can socially integrate into groups of fish.
\end{abstract}

\section{Keywords}

collective behaviour, mixed-groups, biohybrid systems, zebrafish, biomimetic robotics

\section{INTRODUCTION}

Robots are used in ethology and behavioural studies to untangle the multimodal modes of interactions and communication between animals. They can be biomimetic mechanical artefacts that are teleoperated in order to trigger a response from the studied animals. This allows testing various hypothesis on the type of signal used by animals for social interactions [1], [2]. In those studies the human is still in the loop of interactions between the artefact and the animal. This simplifies the design, the implementation and the control of the robot. Alternatively, since the pioneer work of the robot sheepdog and ducks [3], an increasing number of studies [4] have used fully autonomous robots to interact with animals. These robots are programmed to induce reproducible stimuli (possibly embedded in the social context), in order to observe the response of the studied animals during repetitive, sustained and long-lasting social interactions. In this case, the human is not in the experimental loop, and doesn't even need to be present during the experiments. Examples of autonomous robots used in ethology include cows [5], drosophila flies [6] or fish [7]. Different strategies exist to build robot and animal interactions:

(i) First, the artificial systems do not copy any feature of the animal but send cues that the animal responds to. It can for example make use of supra-normal stimuli [8]. The cues can also be abiotic repellent signals such as threats or pain (like electric shocks), or can be attractant such as food or any other attracting chemicals or features. The devices can be carried by animals that are somehow tele-operated by these devices [5]. We call this approach non-biomimetic as the aim of the design of the robots or devices is not to mimic a feature of the studied animal. Indeed, being biomimetic is not a necessity to interact with animals.

(ii) Second, the artificial agent acts as a different animal species such as a sheepdog. The robot can be biomimetic but to another species like a dog for the sheep. This can be a special biomimetic case of inter-species interactions [9] if the robot copies the sheepdog-like animal in its features and behaviours and pertains also to the next category below. But this approach can also be non biomimetic and fall back to the first approach, the robot being just an alien agent interacting with the animals [3].

(iii) Third, the artificial agent is mimicking the animal, luring it as if being the same animal species and using similar signals and behaviours [10]. We call this approach biomimetic and it is the approach developed in this study. 
However, the biomimetic parameter and feature space are very large. The challenge is to choose the most relevant features like some specific visual resemblance, olfactory signature, behavioural similarity. This approach needs metrics to compare each selected features or set of features to select the most relevant ones to avoid the elusive task of building an artificial animal in all its features. We define social integration as being part of a group displaying biomimetic features compared with the animals. The social integration (and its quantification using these metrics) of a robot in a group of fish is the main contribution developed in this study.

Several studies (see Tab. I) have used robots to influence or to control the behaviour of fish, either alone or in a shoal. Most studies involve a robot driven by simple and non-adaptive behaviours (termed fixed pattern in Tab. I), and open-loop social interactions. Closed-loop interactions occur when the robot influences the behaviour of the fish, and the fish influence the behaviour of the robot. The study on the electric fish (Mormyrus rume) opens interesting perspectives where a physical communication signal is used by both the animals and the robots in a closed loop [11]. The electric fish study is closer to the work done on cockroaches where a chemical signal was used [10]. However in [11], the fish robot system represents a breakthrough where a modulated signal is used to achieve a close-loop communication, in this case electric.

Often the robotic systems are composed of two parts. The first part is a robot actuating the second part that is an artefact mimicking some fish features (see Tab. I). This second part acts as a lure to the fish. This lure can be designed to look like a conspecific. In general, the term "robot" is used loosely to mean the whole system or only the actuating first part. Here, we refer to the second part as "the lure" because it carries the bio-mimetic body features.

The studies [12], [13], [14] describe experiments where there is a closed-loop of social interactions and adaptive robotic behaviour. Additionally, [14] presents experiments where the robot was driven by a biomimetic behaviour; but it was not adaptive (with respect to the fish behaviour) and only followed fixed-patterns of behaviour. However, in these studies, the integration of the robot in fish groups is not quantified and not established. Indeed, this kind of controller implies that the robot is more a follower than a real group-member (i.e. integrated into the group) making its own decisions. Simply following the centroid of the group is not biomimetic as fish do not exhibit this kind of behaviour, and such controller does not allow the robot to initiate action but forces it to simply follow fish. In this regard, the embodiment of biomimetic behavioural models could lead to a better integration of artificial agents in animal groups and could allow the robots to influence the collective decision of the mixed group by giving specific preferences to the robot by tuning parameter values of the model [10]. While such controllers have been said to be developed in [13], no experiments have been analysed and reported in the literature yet. Moreover, social integration can only be measured on long-lasting experiments, as it allows to test robot social integration across a large set of social and environmental contexts. While such long-lasting experiments where described in [13] (30 minutes experiments), the non-adaptive biomimetic experiments in [14] were relatively shorter (five minutes experiments).

In this study, we ask the following question: can we quantify and discriminate between sets of biomimetic features needed for a robotic fish to be socially integrated into a group of fish as an autonomous member of the group? We make the hypothesis that this robot must be designed not only to be biomimetic at the level of the lure morphology and other physical aspects but also at the level of its social behaviours.

Here, we propose to implement, in a closed-loop of social interaction, a multi-level and context-dependent biomimetic behavioural model as a controller of a small mobile robot moving a biomimetic fish-lure in a closed tank with a shoal of zebrafish.

We observe ten different groups of four zebrafish and one fish-robot moving in a two-patch square tank. For each trial, we track and identify all agents. Contrary to more cohesive species, the zebrafish often tend to have a very dynamical collective behaviour, with short-lived sub-groups of individuals. Because such splitting influences their behaviour as its changes the social context, we use a simple clustering method to identify sub-groups of agents. Then, we analyse the collective dynamics of the population, and the propensity of the robot to be integrated into the fish group. These results are then compared with data obtained by observing five zebrafish swimming in the same conditions without the fish-robot. In order to compare the experiments with fish and a robot to the control experiments with only fish, we define a similarity measure quantifying the social integration. This measure is based on several metrics characterising the impact of the chosen biomimetic features: the linear speed distribution of the agents, the inter-individual distances distributions of agents in a sub-group, the distribution of sub-group size. 


\begin{tabular}{|c|c|c|c|c|c|}
\hline Type of robots & Study & Robot behaviour & Social Interaction & Number of fish & Type of lure \\
\hline Fixed to & Phamduy et al. 2014 [15] & Fixed pattern & open-loop & Single & Biomimetic \\
\hline \multirow[t]{13}{*}{ mobile arm } & Polverino et al. 2013 [16] & Fixed pattern & open-loop & Shoal & Biomimetic \\
\hline & Polverino \& Porfiri 2013 [17] & Fixed pattern & open-loop & Shoal & Larger Size \\
\hline & Abaid et al. 2012 [18] & Fixed pattern & open-loop & Single & Biomimetic \\
\hline & Butail et al. 2014 [19] & Fixed pattern & open-loop & Single & Biomimetic \\
\hline & Ladu et al. 2015 [20] & Fixed pattern & open-loop & Single & Biomimetic \\
\hline & Ladu et al. 2015 [21] & Fixed pattern & open-loop & Single & Biomimetic \\
\hline & Polverino et al. 2012 [22] & Fixed pattern & open-loop & Shoal & Larger Size \\
\hline & Spinello et al. 2013 [23] & Fixed pattern & open-loop & Single & Larger Size \\
\hline & Polverino \& Porfiri 2013 [24] & Fixed pattern & open-loop & Single & Biomimetic \\
\hline & Ruberto et al. 2016 [25] & Fixed pattern & open-loop & Single & Biomimetic \\
\hline & Bartolini et al. 2016 [26] & Fixed pattern & open-loop & Single & $\begin{array}{l}\text { Biomimetic (several lures } \\
\text { on a robot) }\end{array}$ \\
\hline & Kruusmaa et al. 2016 [27] & Fixed pattern & open-loop & Shoal & Biomimetic (shape only) \\
\hline & Donati et al. 2016 [11] & $\begin{array}{l}\text { Fixed pattern, communication by } \\
\text { electric signals and tail beats }\end{array}$ & closed-loop & Shoal & $\begin{array}{l}\text { Biomimetic (shape and } \\
\text { communication) }\end{array}$ \\
\hline Self- & Abaid et al. 2013 [28] & Fixed pattern & open-loop & Single & Larger Size \\
\hline \multirow[t]{2}{*}{ propelled } & Butail et al. 2013 [29] & Fixed pattern & open-loop & Shoal & Larger Size \\
\hline & Butail et al. 2014 [30] & Fixed pattern & open-loop & Shoal & Larger Size \\
\hline Moved by a & Faria et al. 2010 [7] & Fixed pattern & open-loop & Single & Biomimetic \\
\hline \multirow[t]{3}{*}{ mobile robot } & Swain et al. 2012 [12] & Follow the centroid & closed-loop & Shoal & Biomimetic \\
\hline & Landgraf et al. 2014 [13] & Follow the centroid or Recruitment & closed-loop & Shoal & Biomimetic \\
\hline & Landgraf et al. 2016 [14] & Follow the centroid or Recruitment & closed-loop & Shoal & Biomimetic \\
\hline
\end{tabular}

TABLE I: Recent research using robots to study fish behaviour. We classified the studies according to the technique used to move the lure, the behaviours of the lure, the number of fish swimming with the robot and the shape of the lure. The robots used in these studies can be classified into three categories: First, by using a fish-lure fixed to a mobile arm. This allows to test the reaction of one (or a few) fish to lures that differ by one characteristic, e.g. colour, size, tail-beating frequency. Second, by using a self-propelled aquatic robot that swim in the water with the animals. Such robots are fully autonomous and are a first step towards robots that can be used out of the lab in natural conditions. Third, by using a fish-lure linked through magnets to a mobile robot that moves under the tank. This allows the decoupling of the stimuli shown to the fish (the mock-up fish in the water) and the robot responsible for mobility. By doing so, it is possible to develop a biomimetic lure that has the same size and aspect as a real fish but also capable of reproducing their movement patterns. Four types of lures are used in the literature: biomimetic lures are designed to look like (in term of shape and texture) a fish and are of similar size of a fish; biomimetic (several lures) involves several lures actuated by a single robot; biomimetic (shape only) lures have a biomimetic shape, but not a biomimetic texture; large sized lures are not biomimetic, and are (far) larger than a fish. In [11], the lure is equipped with electric sensors and actuators, which enables the lure to communicate with a shoal of electric fish and to create closed-loop interactions between fish and robot.

\section{Materials AND Methods}

\section{A. Ethics statement}

The experiments performed in this study were conducted under the authorization of the Buffon Ethical Committee (registered to the French National Ethical Committee for Animal Experiments \#40) after submission to the French state ethical board for animal experiments.

\section{B. Experimental set-up}

We use ten groups of five adults wild-type AB zebrafish (Danio rerio) in our experiments.

Our experimental set-up (Fig. 1A) is equipped with two environmental patches (rooms) linked by a corridor (see Fig. 2A). The geometry of the setup is designed to study collective transitions between patches allowing to quantify the group cohesion and collective decision-making as in [31], [32]. Thus the robot has to be socially integrated to be part of the collective transitions between the rooms. The floor of the aquarium is covered with a sheet of teflon to provide a smooth surface for the motion of the fish-lure. An overhead camera (Fig. 1A) captures video frames of experiments. Then, our control and tracking software (called CATS2 [33]) is able to track the positions of the agents (fish and robots), and to control the robots. A system that controls the robots of a mixed-society containing zebrafish must cope with their fast reaction time and sudden movements. Our system is designed to handle low latencies (less than $70 \mathrm{~ms}$, as we work in 15 frames per seconds), both at the tracking and at the control levels. 
The control of fish lure motion is done through events that are sent from the control software and that contain the parameters for the locomotion.
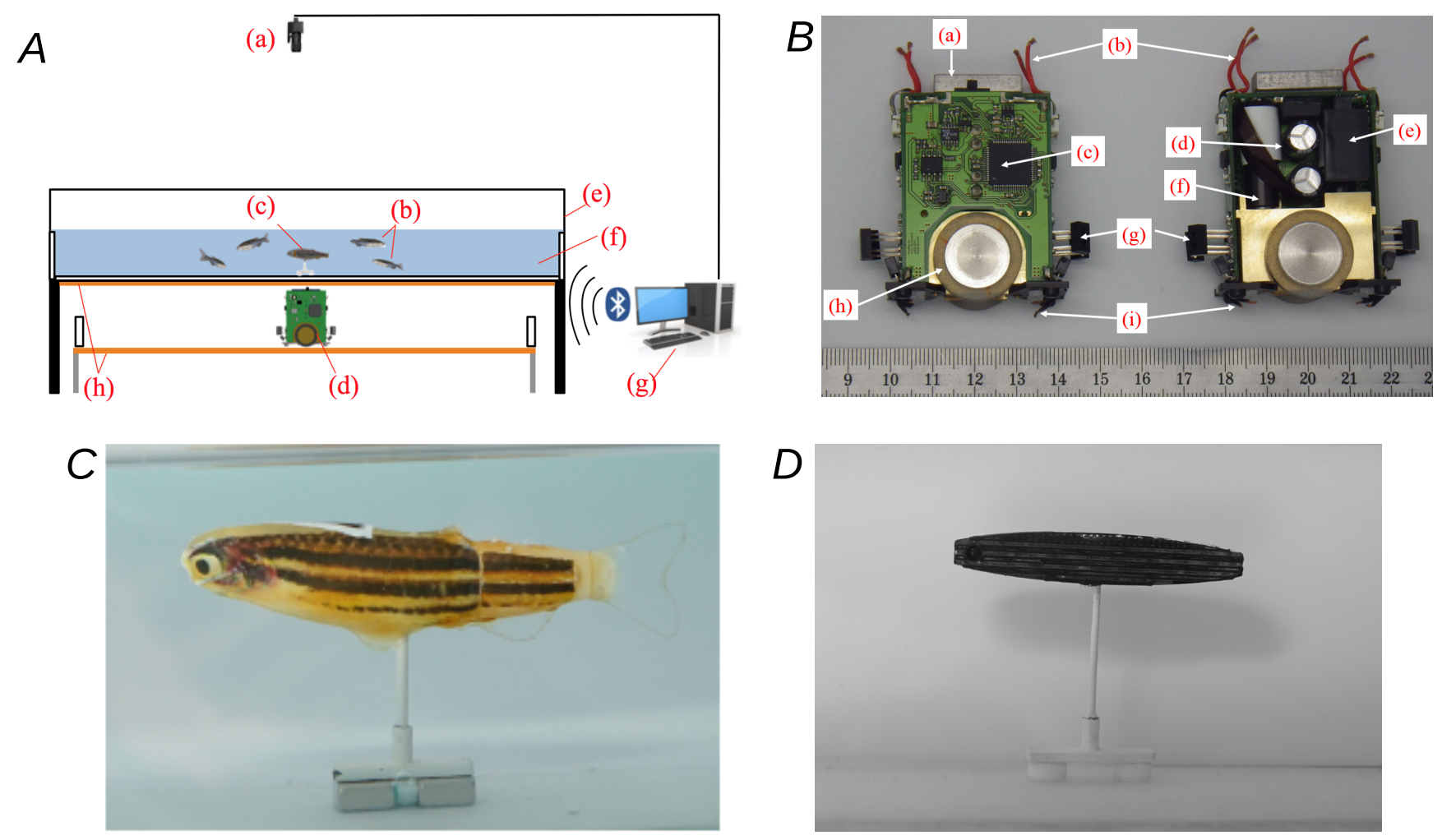

Fig. 1: Panel A: Experimental set-up used during the experiments [34], [35], [36]. (a) Camera used to track the lure and the zebrafish. (b) Zebrafish. (c) Fish-lure inside the aquarium linked to the mobile robot through magnetic coupling. (d) FishBot moving under the aquarium. (e) Aquarium of $1000 \times 1000 \times 250 \mathrm{~mm}$. (f) Water layer of $60 \mathrm{~mm}$ depth. (g) The computer that processes the camera frames and remotely controls the robots via Bluetooth. (h) Conductive plates to power the mobile robot. Panel B: Description of the FishBot [34], [35], [36], the robot used for mimicking fish motion patterns. a) Magnets to magnetically couple FishBot with the lure module. b) Electric brushes to retrieve the power from the positive conductive plate. c) Microcontroller dspic33f128. d) Supercaps that store power if the contact with the plates is lost. e) Bluetooth antenna. f) Maxon DC motor. g) Infrared Proximity sensors. h) Wheel i) Electric brushes to retrieve the power from the Ground connected conductive plate. Panel C: biomimetic lure used during the C1,C2,C3 experiments (see Table III) [34], [35], [36]. Panel D: Non-biomimetic lure used in the $\mathbf{C 4}$ experiment.

\section{FishBot and fish lures}

We consider two kinds of fish lures: a biomimetic lure, and a non-biomimetic lure. The biomimetic lure was designed to mimic the visual appearance of a zebrafish as close as possible (Fig. 1B) using the methodology presented in [37], based on a 3D scan of a zebrafish. The lure was also covered with a decal to have the similar color patterns as the zebrafish (the methodology is described in Supplementary Information). The non-biomimetic lure is a zebrafish-sized 3D printed black ellipsoid (of $4.5 \times 0.5 \times 0.8 \mathrm{~cm}$ ). The lures are linked by magnetic coupling to a mobile robot moving below the experimental tank.

We use the miniature mobile robot "FishBot" described in [34], [35], [36] that can achieve the required speeds and accelerations in order to reproduce the fish displacement under water. The robot is continuously powered as described in Figs. 1A and 1C and controlled with a wireless bluetooth link, therefore it is possible to achieve long duration experiments in closed-loop (described in Supplementary Information). 


\section{Data analysis}

Zebrafish tend to form short-lived sub-groups of individuals (especially in fragmented environments [31]), which often translate to different sub-group behaviour, as sub-group size changes the social context. In particular, fish do not behave the same way when they are alone as when they are in a group (for instance, they have different distributions of linear and angular speeds). As such, we identify sub-groups of agents in each frame of our experimental videos. We use a clustering algorithm that uses only the position of each individual to detect the sub-groups in each frame of our experimental videos. This algorithm is described in Supplementary S1.

Then, by using the tracked positions of agents (fish and robot) and the information describing sub-groups membership, we compute several statistics of individual and collective behaviour: the density of presence in the arena, the distributions of linear speeds in each room, the inter-individual distances in sub-groups of size two to five individuals, and the distribution of the number of agents in sub-groups.

\section{E. Quantifying social integration}

To quantify the social integration of the robot into the group of fish, we compare the results of the experiments with a robot with the corresponding results from the biological reference case. We define a similarity measure to compare the results of all experiments, taking into account the distribution of linear speeds, the distribution of inter-individual distances and the distribution of sub-groups size. We postulate that this similarity measure translates directly to the capabilities of the robot to socially integrate into the group of fish. We term this similarity measure the social integration index $(\mathrm{I}(\mathrm{Ci})$ for the $\mathrm{Ci}$ experiment), and define it as:

$$
\begin{gathered}
I(C i)=\frac{I_{\text {linSpeedRooms }}(C i)+I_{\text {linSpeedCorridor }}(C i)+I_{\text {interindivSG2 }}(C i)+\ldots+I_{\text {interindivSG5 }}(C i)+I_{\text {SGSize }}(C i)}{7} \\
I_{\mathrm{a}}(C i)=1-H\left(C 0_{\mathrm{a}}, C i_{\mathrm{a}}\right)
\end{gathered}
$$

The social integration index $I(\mathrm{Ci})$ of the experiment $C i$ has a value between 0.0 and 1.0. A value of 0.0 corresponds to the absence of social integration of the robot, and a value of 1.0 corresponds to a social integration of the robot comparable to that of a fish.

The vectors $I_{\text {linSpeedRooms }}(C i), I_{\text {linSpeedCorridor }}(C i), I_{\text {interindivSG2 }}(C i), \ldots, I_{\text {interindivSG5 }}(C i)$ and $I_{\text {SGSize }}(C i)$ correspond respectively to the histograms of the distributions of linear speeds in the rooms, linear speeds in the corridor, interindividual distances in sub-groups of sizes two to five individuals and to the size of sub-groups, for experiment $C i$. These histograms are presented in Figs. 6, 7 8, and in Supplementary Information. We hypothesise that these are the most relevant features to identify the social integration of the robot into the group of fish. First, the speed distributions show the ability of the robot to follow the fish at the same speed as the fish. Second, the inter-individual distance distributions show the capability of the robot to be at the same distance to the fish as a fish would be towards its neighbours. Lastly, the distribution of sub-group sizes shows that sub-group dynamics in experiments with a robot are similar to those in experiments with only fish.

The $H(P, Q)$ function is the Hellinger distance between two histograms. The Hellinger distance [38] is a divergence measure, similar to the Kullback-Leibler (KL) divergence. However, the Hellinger distance is symmetric and bounded, unlike the KL-divergence (and most other statistical distance metrics). It is often used as a metric to compare distributions, and is defined as:

$$
H(P, Q)=\frac{1}{\sqrt{2}} \sqrt{\sum_{i=1}^{d}\left(\sqrt{p_{i}}-\sqrt{q_{i}}\right)^{2}}
$$

where $p_{i}$ and $q_{i}$ are respectively the $i$-th bin values of histograms $P$ and $Q ; d$ is the number of bins in both histograms $P$ and $Q$. 

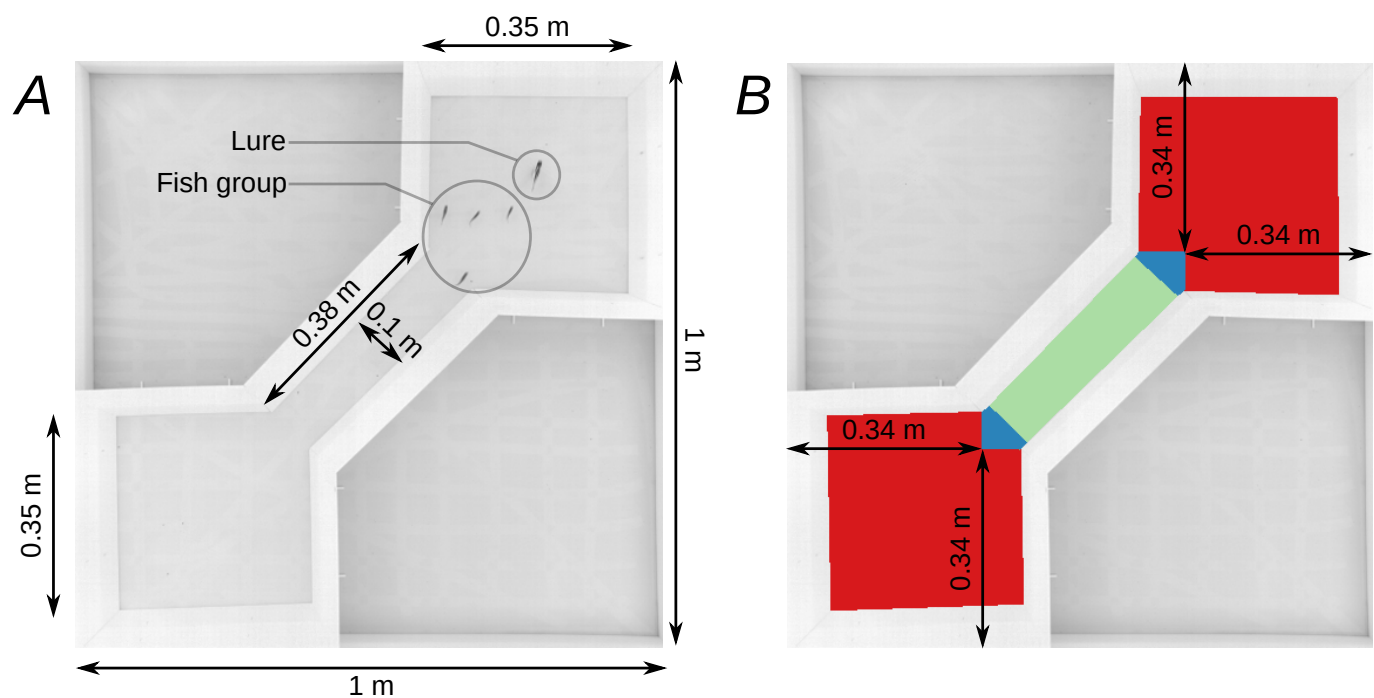

Fig. 2: Panel A: Experimental arena composed of two linked square rooms used to study collective transitions and decision-making. Our biomimetic lures, described in Fig. 1B, can be integrated within the fish group and mimic their collective behaviours. Panel B: Colored zones of the arena corresponding to the three different types of behaviour of the robot. These behaviours are outlined in Table II. When the robot is in the rooms (in red) or near the entrance of the corridor (in blue), it is driven by the biomimetic model presented in Sec. III. This model is used to generate a new target position of the robot every $1 / 3 \mathrm{~s}$. If this target position is near the entrance of the corridor (in blue), the target position of the robot is not updated before $5 \mathrm{~s}$ are passed, to give the robot enough time to go to the entrance of the corridor. When the robot is in the corridor (green zone), it passes straight through the corridor, with a constant speed.

\begin{tabular}{|c|c|c|c|c|c|}
\hline Color in Fig.2B & $\begin{array}{l}\text { Robot Current } \\
\text { Zone }\end{array}$ & Target Zone & Trajectories & $\begin{array}{l}\text { Movement } \\
\text { patterns }\end{array}$ & Obstacle avoidance \\
\hline Red & $\begin{array}{l}\text { In Rooms or } \\
\text { Near entrance }\end{array}$ & In Rooms & $\begin{array}{l}\text { probabilistic (cf. Sec. III-A), } \\
\text { updated every } 1 / 3 \mathrm{~s}\end{array}$ & $\begin{array}{l}\text { biomimetic } \\
\text { (cf. Sec. III-B) }\end{array}$ & Turn to avoid (cf. Sec. III-B) \\
\hline Blue & $\begin{array}{l}\text { In Rooms or } \\
\text { Near entrance }\end{array}$ & Near entrance & $\begin{array}{l}\text { probabilistic (cf. Sec. III-A), } \\
\text { updated every } 5 \mathrm{~s}\end{array}$ & $\begin{array}{l}\text { biomimetic } \\
\text { (cf. Sec. III-B) }\end{array}$ & Turn to avoid (cf. Sec. III-B) \\
\hline Green & Corridor & any & Pass-through (cf. Sec. III-C) & $\begin{array}{l}\text { Constant speed } \\
\text { (cf. Sec. III-D) }\end{array}$ & Braitenberg (cf. Sec. III-D) \\
\hline
\end{tabular}




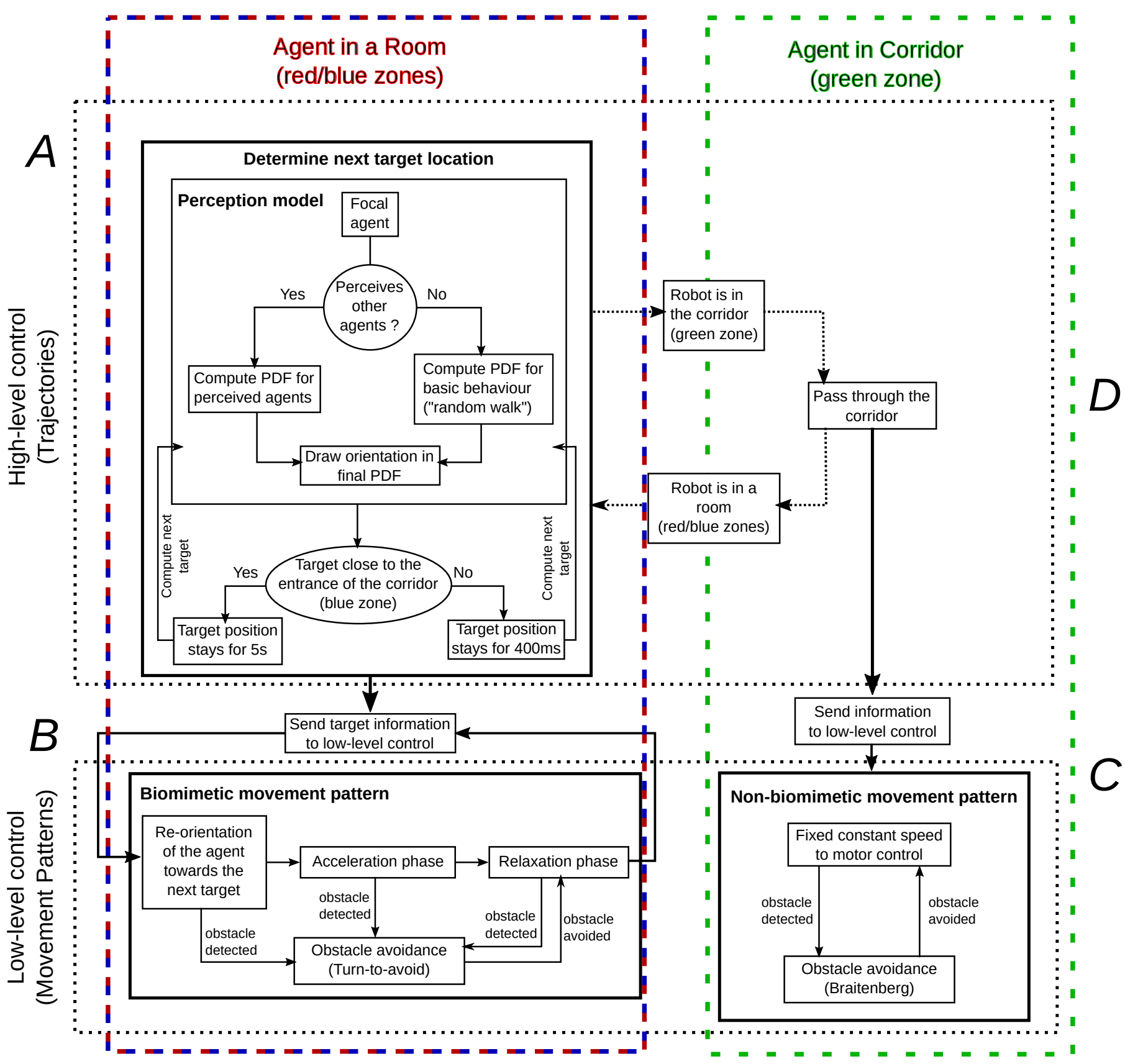

Fig. 3: Multilevel behavioural model (here denoted BM) used as robot controller. Our robot control system is divided into two layers. The high-level control layer (in A and D) describes the trajectory of the robot in the arena and generates target positions. The low-level control layer (in B and C) handles the movement patterns of the robot to reach the target positions derived from the high-level control layer. The robot has different behaviours depending on whether it is in the rooms (in A and B, represented in red and blue in Fig. 2B) or in the corridor (in $\mathrm{C}$ and $\mathrm{D}$, in green in Fig. 2B). In the rooms, the high-level (in A) and low-level (in B) controls are biomimetic. In the corridor, the high-level (in D) and low-level controls (in C) are not biomimetic, and drive the robot to transit from one room to the other through the corridor. These behaviours are summarised in Table II. 


\section{MULTI-LEVEL APPROACH FOR THE ROBOT BEHAVIOUR}

We present here a biomimetic model of fish behaviour, that can be implemented as a robotic controller. Our model is multi-contextual, to take into account the different behaviours exhibited by the fish in the different zones of our fragmented experimental set-up (with two rooms and a corridor, cf Fig. 2A). We design this model to be multi-level (Fig. 3.

The high-level control manages the biomimetic trajectories of the robot. We use different behavioural schemes to generate desired target positions of the robot depending on its position in the arena. These trajectories are calibrated to correspond to the analysed fish trajectories.

The low-level control corresponds to the movement patterns of the robot that are also important to facilitate social integration. It describes how the robot can move from its current position to the target positions provided by the high-level control system.

Control at both levels can be designed to be biomimetic. At the level of trajectories, zebrafish have a complex social behaviour and tend to form dynamic and short-lived groups. At the level of movement patterns, zebrafish move by successive bouts of tail-beats [39]. Each tail-beat can be modelled as a sequence of three steps: first, the tail-beat allows the fish to reorient itself towards its target position; second, the fish accelerates linearly by using the thrusting effect of the tail-beat; third, the tail-beating stops and the fish slides into water with a decreasing linear speed [36]. Here, we present a biomimetic multi-level model of these dynamics, taking into account both high-level (in Sec. III-A) and low-level (in Sec. III-B) controls.

Figure 2B and Table II summarise the different behaviours depending of the position of the robot, and how they are implemented in terms of high-level control (trajectories) and low-level control (movement patterns). A general description of this biomimetic model can be found in Fig. 3.

We designed our behavioural model to exhibit different behaviours depending on the spatial position of the robot in the arena. Indeed, we observed that zebrafish exhibit different behaviours when they are in the corridor compared to when they are in a room [31] (cf Supplementary Information Figs. S2, S3, S4, S5). When they are in the corridor, they tend to go from one of the entrances of the corridor to the other room, with a different speed distribution. The fish only transit in the corridor in a rather straight manner.

When the robot is in the rooms (in red) or near the entrance of the corridor (in blue), it is driven by a biomimetic probabilistic behavioural model (Fig. 3A), and described below (cf Sec. III-A). This model is used to generate new target positions of the robot every $1 / 3 \mathrm{~s}$ (this time step was chosen to correspond to the tail beat frequency of the zebrafish of $\sim 2.5 \mathrm{~Hz}$, as in [40]). The low-level controller of the robot makes it follow and reach these target positions with fish-like biomimetic movement patterns (Fig. 3B, cf Sec. III-B). If a target position of the robot is near the entrance of the corridor (in blue), it is not updated before $5 \mathrm{~s}$ are passed, to give the robot enough time to reach the entrance of the corridor. When the robot is in the corridor (in green), it passes straight through the corridor with a constant speed.

At present, while the robot can pass through the corridor from one room to the other without wall-collision, it can be difficult for the robot to perform U-turns in the corridor (i.e. turn around in the corridor and go back to the room the robot was previously situated) without colliding into the walls due to the narrowness of the corridor. As such, when the robot enters the corridor, it is driven by a pass-through behaviour to transit from one room to the other in a straight line, without possibility of U-turn (Fig. 3D). We do not use the biomimetic probabilistic behavioural model (Fig. 3A) in this case, as it would have a small probability of generating target positions toward the room where the robot was previously situated, thus driving the robot to perform an U-Turn.

Additionally, the teflon sheet that we put on the floor of the aquarium is not perfectly plane (as it is difficult to glue this layer to the aquarium), which can slightly influence the movement of the robot due to the magnetic coupling between the robot and the lure (sliding on the teflon layer). This is especially the case when the robot is moving at high speed, or when it performs strong accelerations, for instance when it is driven by the biomimetic low-level controller (Fig. 3B). This is why we use a constant speed behaviour (without strong acceleration, Fig. 3C) when the robot is passing through the corridor.

\section{A. High-level biomimetic behavioural model in the rooms}

To define the trajectories of the robot in the rooms (red and blue zones in Fig. 2B, Fig. 3A), we developed a biomimetic behavioural model inspired from the probabilistic model in [40], which used a gaussian mixture agentbased approach to describe zebrafish behaviour in a shoal. However, the model in [40] was designed to model 
zebrafish behaviour in an empty square arena. We modified this model to be used as a robotic controller, and to handle our arena with two rooms and a corridor.

In this model, the agents update their position vector $X_{i}$ with a velocity vector $V_{i}$ :

$$
\begin{gathered}
X_{i}(t+\delta t)=X_{i}(t)+V_{i}(t) \delta t \\
V_{i}(t+\delta t)=v_{i}(t+\delta t) \Theta_{i}(t+\delta t)
\end{gathered}
$$

The speed $v_{i}$ is drawn from the speed distribution of the fish in the rooms (cf Supplementary Information Fig. $\mathrm{S} 4$, experiment $\mathrm{C} 0$ ). The orientation $\Theta_{i}$ is drawn from the probability density function (PDF) computed as a mixture distribution of von Mises distributions centred on the stimuli perceived by the focal agent. In this study, we only take into account the influence of other agents. The attraction towards the walls is not considered yet as it would put the robots too close to the walls, increasing greatly the number of collisions between them.

The PDF $f_{0}(\theta)$ for an agent to move in each potential direction $\theta$ in a bounded environment without perceptible stimulus is given by:

$$
f_{0}(\theta)=\frac{\exp \left(\kappa_{0} \cos (\theta)\right)}{2 \pi I_{0}\left(\kappa_{0}\right)}
$$

with $\kappa_{0}$ a dispersion parameter associated with movements not influenced by stimulus, and $I_{0}$ the modified Bessel function of first kind of order zero, defined as:

$$
I_{0}(\kappa)=\sum_{k=0}^{\infty} \frac{(\kappa / 2)^{2 k}}{k ! \Gamma(k+1)}
$$

where $\Gamma(z)$ is the gamma function:

$$
\Gamma(z)=\int_{0}^{\infty} x^{z-1} e^{-x} d x
$$

The model computes a PDF for the focal agent to move according to other agents (congeners). The probability of the focal agent to orient towards a perceived agent is given by a von Mises distribution clustered around this agent:

$$
f_{f_{i}}=\frac{\exp \left(\kappa_{f} \cos \left(\theta-\mu_{f_{i}}\right)\right)}{2 \pi I_{0}\left(\kappa_{f}\right)}
$$

with $\theta$ the potential direction of movement of the agent, $\mu_{f_{i}}$ the location of the perceived fish $i$ and $\kappa_{f}$ a dispersion parameter associated with agent attraction.

The model computes a weighted sum of all distributions $f_{f_{i}}$ for all agents $i$, resulting in a PDF $f_{F}(\theta)$ defined as:

$$
\begin{gathered}
f_{F}(\theta)=\sum_{i=1}^{n_{f}} \frac{A_{f_{i}}}{A_{T_{f}}} \frac{\exp \left(\kappa_{f} \cos \left(\theta-\mu_{f_{i}}\right)\right)}{2 \pi I_{0}\left(\kappa_{f}\right)} \\
A_{T_{i}}=\sum_{i=1}^{n_{f}} A_{f_{i}}
\end{gathered}
$$

with $A_{T_{f}}$ the sum of solid angles $A_{f_{i}}$ captured by each perceived agent $i$ and $n_{f}$ the number of perceived agents.

Finally, we calculate a weighted sum of the PDFs to obtain the global probability distribution function $f(\theta)$ of the focal fish to move towards a given direction. This global PDF is different from [40], as we removed the attraction to the walls, and is computed as follow:

$$
f(\theta)=\frac{f_{0}(\theta)+\alpha_{0} A_{T_{f}} f_{F}(\theta)}{1+\alpha_{0} A_{T_{f}}}
$$

with $\alpha_{0}$ a parameter weighting the influence of the perceived agents. The parameters $\kappa_{0}=6.3$ and $\kappa_{f}=20$ are the same as in [40].

There is a delay $(<500 \mathrm{~ms})$ between the time when a new target position is computed and the time when the robot actually reaches this position. This can have an adverse effect on the desired fish-following behaviour of the model, when the robot is part of a sub-group and is moving alongside its neighbours. Indeed, the target-following system (low-level control) must compromise between efficiency (the capability of reaching the target with a low 
latency) and biomimetism (fish-like movement, and fish-like speed distribution). To mitigate this effect, we selected a different value of $\alpha_{0}$ than in [40]: we consider $\alpha_{0}=n_{A} * 1000 / n_{D}$ with $n_{A}=5$ the total number of agents in the experiment, and $n_{D}$ the number of detected agents in the current frame. This increases the tendency of the robot to follow fish groups. The number of detected agents is used to normalise the computation of $\alpha_{0}$ as the model only takes into account the agents that are actually detected by the tracking system, which introduces a bias.

Then, we numerically compute the cumulative distribution function (CDF) corresponding to this custom PDF $f(\theta)$ by performing a cumulative trapezoidal numerical integration of the PDF in the interval $[-\pi, \pi]$ (as described in [40]). Finally, the model draws a random direction $\Theta_{i}$ in this distribution by inverse transform sampling. The position of the fish is then updated according to this direction and his velocity with equations 4 and 5 . If the target position of the robot is in an unreachable area (e.g. the walls), we draw another random direction from the CDF.

\section{B. Low-level biomimetic movement patterns in the rooms}

In the rooms (red and blue zones in Fig. 2B), the robot is following trajectories drawn by the high-level biomimetic model described in Sec. III-A. The low-level robot controller (Fig. 3B) is programmed to follow the target position computed using the high-level behavioural model. When the target is near the entrance of the corridor (blue zone of Fig. 2B), it remains there for $5 s$ to leave enough time for the robot to reach the entrance of the corridor, and then pass through it to the next rooms. We choose the relatively long duration of $5 s$ to prevent the high-level model to generate a new target not situated in the corridor before the robot is able to reach the entrance of the corridor, as it could lead to experiments where the robot never go through the corridor.

To accurately mimics the movement patterns of the zebrafish inside the two rooms, we used the low-level robot controller designed in [36]. At each control steps, the desired target position generated by the model described in Sec. III-A is compared with the current robot position and orientation. The difference in orientation is sent to the robot, that executes a step machine composed of three steps: First, the wheels are controlled in position to reorient the robot towards the desired target (Re-orientation phase). Second, the robot accelerates to $0.7 \mathrm{~m} . \mathrm{s}^{-2}$ (Acceleration phase) and third, the speed of the robot is set to a constant speed of $8.5 \mathrm{~cm} \cdot \mathrm{s}^{-1}$ (Relaxation phase). The robot will keep this speed until the next control step. This mimics the typical locomotion of zebrafish in open areas as demonstrated in [36].

We use a simple collision avoidance scheme (termed Turn to avoid), where the robot stops when it is too close to a wall and then turns at a reduced speed $\left(5 \mathrm{~cm} . \mathrm{s}^{-1}\right)$ for $3.5 \mathrm{~s}$ before going back to its normal behaviour.

\section{Robot trajectories in the corridor}

When the robot is in the corridor (green zone in Fig. 2B), it is programmed to transit in a straight line towards the other room (Fig. 3D), with a constant speed of $12 \mathrm{~cm} \cdot \mathrm{s}^{-1}$ if the robot is not in collision with a wall. This speed is reduced to $5 \mathrm{~cm} . \mathrm{s}^{-1}$ when the robot is too close to the walls, e.g. after a collision. The resulting mean speed of the robot is still lower than the one of the fish (cf Supplementary Information). We could not increase the speed of the robot to match the mean speed of the fish in the corridor, as it would increase the probability of crashing against a wall.

\section{Biomimetic movement patterns in the corridor}

Inside the corridor (green zone in Fig. 2B), the zebrafish usually have a constant speed as they are stressed due to the reduced size of the environment. Therefore, we implemented a controller (Fig. 3C) for the robot to maintain a constant linear speed of $9.4 \mathrm{~cm} . \mathrm{s}^{-1}$ inside the corridor while the rotating speed is controlled using a PID (Proportional-Integral-Derivative) controller for the robot to reach the opposite room.

To mitigate the effect of eventual collisions of the robot with the walls, the low-level controller of the robot implements a Braitenberg-based obstacle avoidance scheme [41]. While this Braitenberg obstacle avoidance scheme is more efficient than the Turn to avoid scheme used in the rooms, it only works when the robot is moving with a constant speed. This is why we did not use it in the rooms, where the robot is driven by the biomimetic movement patterns (described in Sec. III-D). 


\begin{tabular}{|c|c|c|c|c|c|}
\hline Label & Experiment & Number of agents & Robot Trajectories in the rooms & $\begin{array}{l}\text { Robot Movement pattern } \\
\text { in the rooms }\end{array}$ & Lure \\
\hline $\mathrm{CO}$ & wild-type zebrafish only & 5 fish +0 robot & - & - & - \\
\hline $\mathrm{C} 1$ & Biomimetic model with Biomimetic lure & 4 fish +1 robot & Biomimetic (cf Sec. III-A) & Biomimetic (cf Sec. III-B) & Biomimetic \\
\hline $\mathrm{C} 2$ & Random walk with Biomimetic lure & 4 fish +1 robot & Random Walk & Biomimetic (cf Sec. III-B) & Biomimetic \\
\hline $\mathrm{C} 3$ & Biomimetic model with Constant Speed & 4 fish +1 robot & Biomimetic (cf Sec. III-A) & Constant Speed & Biomimetic \\
\hline $\mathrm{C} 4$ & Biomimetic model with non-biomimetic lure & 4 fish +1 robot & Biomimetic (cf Sec. III-A) & Biomimetic (cf Sec. III-B) & Non-biomimetic \\
\hline
\end{tabular}

TABLE III: Analysed experiments. The CO experiment is the biological reference case, involving a group of five wild-type zebrafish and no robot. The $\mathbf{C 1}$ experiment involves four wild-type zebrafish and one robot driven by the multi-level biomimetic model presented in Sec. III. These experiments are performed in the set-up described in Fig. 1A with the arena in Fig. 2B after a 30 minutes period of acclimatization. Each experiment is repeated ten times and lasts 30 minutes. The $\mathbf{C 2 , C 3}$ and $\mathbf{C 4}$ experiments are control experiments based on the $\mathbf{C 1}$ experiment. The $\mathbf{C 2}$ experiment involves a robot driven by a random walk behaviour, to assess to impact of biomimetic trajectories on social integration. In the $\mathbf{C} \mathbf{3}$ experiment, the robot moves with a constant speed along its trajectory instead of moving using biomimetic movement patterns. The $\mathbf{C} 4$ experiment is performed with a non-biomimetic lure.

\section{RESUlts}

Our goal is to socially integrate a robot driven by a multi-level biomimetic behaviour into a group of laboratory wild-type zebrafish. The experiments are done in a two connected room aquarium that is designed to study social cohesion, collective departures and decision-making [31], [32]. We consider five different experimental conditions summarised in Table III. Each one of the five experimental conditions is composed of ten repetitions of 30 minutes lasting trials. Each experimental condition has thus been tested for five hours in total. We tested ten different fish groups per experimental conditions, i.e. 40 different fish with the robot. We compare these five experiments by using the similarity measure described in Sec. II-E.

\section{A. Individual trajectories}

We rebuild the trajectories of each individual (fish and robot) by using the individual tracking of the agents (cf Sec. II-D). Figure 4 presents examples (of 1 minute) of such trajectories for experiments C0 and C1 (in Panel B, the robot trajectories are in black). The fish tend to follow walls rather than staying in the center of the rooms.

Even though we did not implement any wall following behaviour into the high-level part of the BM model (Fig. IIIA, see Sec. III-A), the robot still exhibits a wall following behaviour as it tends to be attracted to fish. The trajectories of the robot appear qualitatively biomimetic. We further quantify the mixed group in the following sections.
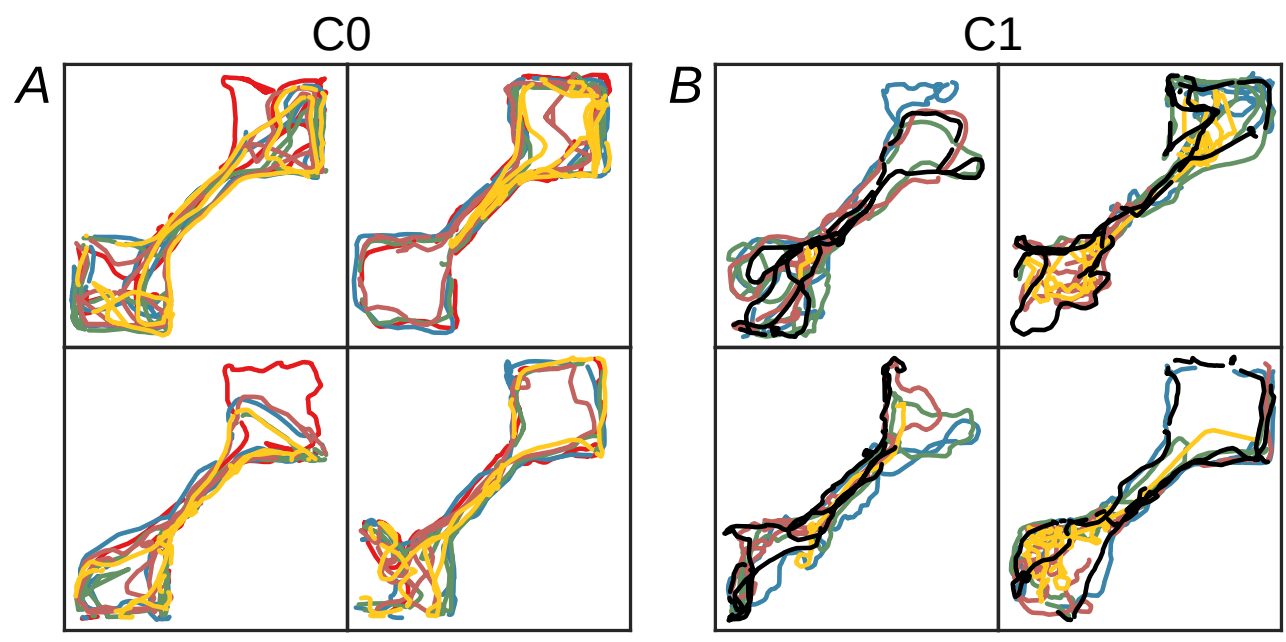

Fig. 4: Examples of trajectories of the agents in experiments $\mathbf{C O}$ (Panel A) and in experiment $\mathbf{C 1}($ Panel B). Each subplot corresponds to 1-minute sections of a 30-minutes experiment. In Panel B, the robot trajectories are in black. 


\section{B. Group clustering and social cohesion}

We compute the mean inter-individual distances between each pair of agents (fish and robots) of a sub-group for all experiments. Three characteristic cases were considered: all sub-groups (i.e. sub-groups of two to five individuals) in Fig. 6, sub-groups of two individuals in Fig. 7, and sub-groups of all (five) individuals in Fig. 8. For all three cases, the distributions of inter-individual distances of the $\mathrm{C} 1$ and $\mathrm{C} 3$ experiments are the closest to the ones of the $\mathrm{C} 0$ experiment.

We compute the mean fraction of non-isolated individuals in each experiment (Fig. 5A). In experiments with a robot, $\mathrm{C} 1$ (mean of $78.1 \%$, std of 0.050 ) is the closest to $\mathrm{C} 0$ (mean of $83.3 \%$, std of 0.037 ), followed by C3 (mean of $75.9 \%$, std of 0.049 ), C4 (mean of $74.4 \%$, std of 0.622 ), and C2 (mean of $67.4 \%$, std of 0.062 ). The robot tends to be isolated slightly more often than the fish. When the robot follows our biomimetic model, it increases its capability to be in a sub-group (experiments $\mathrm{C} 1$ and $\mathrm{C} 3$ ). The type of movement pattern and lure also affect the capability of the robot to be in a sub-group (experiments $\mathrm{C} 2$ and $\mathrm{C} 4$ ).

Figure 5B presents the mean fraction of the population either isolated ("sub-group" of size 1) or in a sub-group with two to five individuals. Agents in the experiments with the robot $(\mathrm{C} 1, \mathrm{C} 2, \mathrm{C} 3, \mathrm{C} 4)$ have a higher probability to be isolated and a lower probability to be in sub-groups of five individuals (whole population). Experiments $\mathrm{C} 1$ and $\mathrm{C} 3$ provide the results that are the most similar to $\mathrm{C} 0$. Indeed, while the robot can integrate into the group of fish, especially when driven by a biomimetic model, its behaviour could still be improved to be closer to a fish.

\begin{tabular}{|c|cccc|}
\hline & C1 & C2 & C3 & C4 \\
\hline Linear speed in the rooms & 0.706 & $\mathbf{0 . 7 3 9}$ & 0.708 & $\mathbf{0 . 7 3 9}$ \\
Linear speed in the corridor & $\mathbf{0 . 8 1 2}$ & 0.694 & 0.608 & 0.637 \\
Inter-indiv. distances in SG of 2 indiv. & $\mathbf{0 . 8 9 4}$ & 0.893 & 0.780 & 0.524 \\
Inter-indiv. distances in SG of 3 indiv. & 0.927 & 0.897 & $\mathbf{0 . 9 7 2}$ & 0.885 \\
Inter-indiv. distances in SG of 4 indiv. & 0.915 & 0.837 & $\mathbf{0 . 9 5 3}$ & 0.798 \\
Inter-indiv. distances in SG of 5 indiv. & $\mathbf{0 . 8 6 6}$ & 0.759 & 0.861 & 0.706 \\
Distribution of SG size & $\mathbf{0 . 8 9 9}$ & 0.854 & 0.875 & 0.848 \\
\hline Social integration index (Mean score) & $\mathbf{0 . 8 6 0}$ & 0.810 & 0.822 & 0.734 \\
\hline
\end{tabular}

TABLE IV: Social integration indexes for all experiments. Higher values correspond to better integration of the robot in the group of fish. Results in bold correspond to the highest values for each feature. The C1 experiment involves 4 wild-type zebrafish and one robot driven by the multi-level biomimetic model presented in Sec. III. The $\mathbf{C 2 , C 3}$ and $\mathbf{C 4}$ experiments are control experiments based on the $\mathbf{C 1}$ experiment. The $\mathbf{C 2}$ experiment involves a robot driven by a random walk behaviour, to assess to impact of biomimetic trajectories on social integration. In the $\mathbf{C 3}$ experiment, the robot moves in a constant speed along its trajectory, instead of biomimetic movement patterns. The C4 is performed with a non-biomimetic lure.
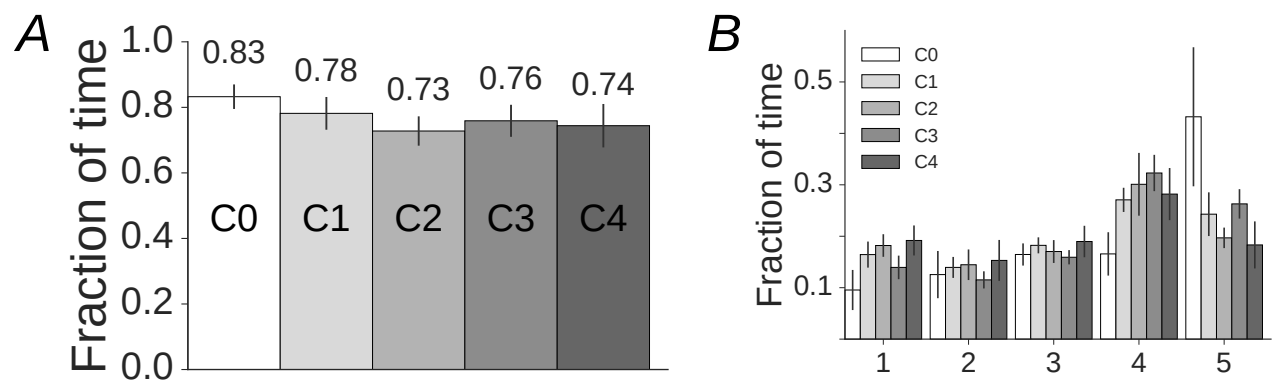

Fig. 5: Panel A: Mean fraction of non-isolated individuals in all experiments. The distributions differ significantly (Two-sample Kolmogorov-Smirnov test, with p-values < 0.05; cf Sec. II-D). Panel B: Mean fraction of the population in a sub-group of one (isolated individuals) to five (whole population) individuals. The distributions differ significantly (Two-sample Kolmogorov-Smirnov test, with p-values < 0.05; cf Sec. II-D). Results are obtained in ten trials of 30 minutes experiments using groups of wild-type zebrafish moving in the set-up described in Fig. 2A. 


\section{Quantifying social integration}

Table IV presents the resulting social integration index and the associated $I_{\mathrm{a}}(\mathrm{Ci})$ values for all experiments with a robot $(\mathbf{C 1}, \mathbf{C 2}, \mathbf{C 3}, \mathbf{C 4})$. The experiment with the highest number of biomimetic characteristics, $\mathbf{C 1}$, has the highest integration index: 0.860. Experiment $\mathrm{C} 3$ has the second highest integration index: 0.822 , followed by $\mathrm{C} 2(0.810)$ and C4 (0.734). It shows the veracity of our hypothesis: a robotic fish has higher social integration capability into a group of fish if it is designed to be biomimetic on three key aspects: the morphology of the lure, the type of trajectories, and the type of movement patterns. Here, we show that the morphology of the lure has the highest impact on social integration, followed by the type of trajectory of the robot and by the type of movement patterns of the robot, with differences of social integration indexes (mean score) with $\mathrm{C} 1$ of respectively $0.126,0.050$ and 0.038 . While $\mathrm{C} 1$ has the highest social integration index, $\mathrm{C} 2$ and $\mathrm{C} 4$ have the highest score of linear speed in the rooms, and $\mathrm{C} 3$ has the highest score in inter-individual distances in sub-groups of three to four individuals.

\section{CONCLUSiONS}

In this study, we present a biomimetic model of fish behaviour in a fragmented environment. This model is multicontextual spatially (square rooms and corridor), multi-level (collective dynamics, collective departures, trajectories, movement patterns) and probabilistic. Then, we describe a methodology to create a closed-loop of social integration between a shoal of zebrafish and a biomimetic fish-lure moved by a robot driven by this biomimetic behavioural model. We use a similarity measure as a metric to quantify the capability of this lure to be socially integrated in the shoal of fish and the biomimetism of the behaviour of the robot.

While previous studies of the literature (Tab. I) showed that a robotic fish needs to be biomimetic in order to be socially integrated in a group of fish, most efforts were made on finding attractive biomimetic lure morphologies, with no or few considerations ([12], [13], [14]) on the effects of robot behaviour (trajectories and movement patterns) on social integration. Both [13] and [14] present experiments involving a closed-loop of interaction between a group of fish and a robot driven by a controller inspired from fish behaviour and magnetically coupled with a biomimetic lure. However, in these studies, the social integration of the robot is not quantified and the robot is more a follower or initiator following fixed patterns of behaviour rather than an entity capable of being integrated with the group of fish and initiating its own decisions during trials lasting 30 minutes.

The biomimetic model of fish behaviour we present can effectively socially integrate a group of wild-type zebrafish.

This problem is challenging because zebrafish present loose social organisation [31]. Indeed this fish species does not form stable schooling patterns (i.e. fish aligned and swimming together in the same direction) but form shoals (tendency to form irregular groups). Moreover, it is difficult to model zebrafish collective behaviour and translate it directly into a robotic controller. Here we consider complex social behaviour in a designed set-up to study social cohesion and collective departures. This fragmented set-up induce more elaborate behaviours than simpler round or square empty tanks. Such environment requires to develop context dependent collective behaviour models taking into account spatial context and social effects.

In this study, we compare mixed groups of one robot and four fish to groups of five fish and no robot. It allows us to quantify the social integration of the robot i.e. if the robot belongs to the group, like another fish, for trials lasting for 30 minutes each and during five hours in total. We present metrics that quantify the distance between a mixed and a pure fish group. According to these metrics, the closer we are to a pure fish group the more the robot is socially integrated.

We analyse the impact of both lure morphology and robot behaviour aspects on social integration and show that a robotic fish driven by a biomimetic behavioural model is more akin to be socially accepted by the fish compared to a robotic fish driven by a simple non-biomimetic behavioural model. We assess the importance of biomimetism on different aspects of the robot design: the morphology of the lure, the type of trajectories of the robot, and the type of movement patterns exhibited by the robot, its decision making capabilities depending on the context. All three aspects are shown to be relevant to facilitate the social integration of the robot. We present results showing that both the lure and the behaviour of the robot are important in the design of robots able to socially integrate a group of fish. We show that the morphology of the lure is the feature with the highest impact, followed by the type of robot trajectories and the type of robot movement patterns.

Additionally, we include a behavioural analysis of the fish group and of the mixed-group of fish and robot. This analysis takes into account the tendency of fish to gather in short-lived and dynamic sub-groups, that can exhibit 
CO

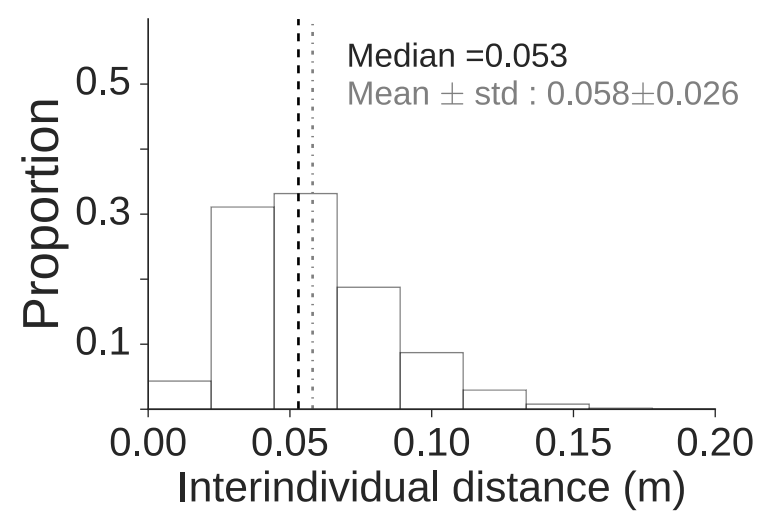

$\mathrm{C} 2$

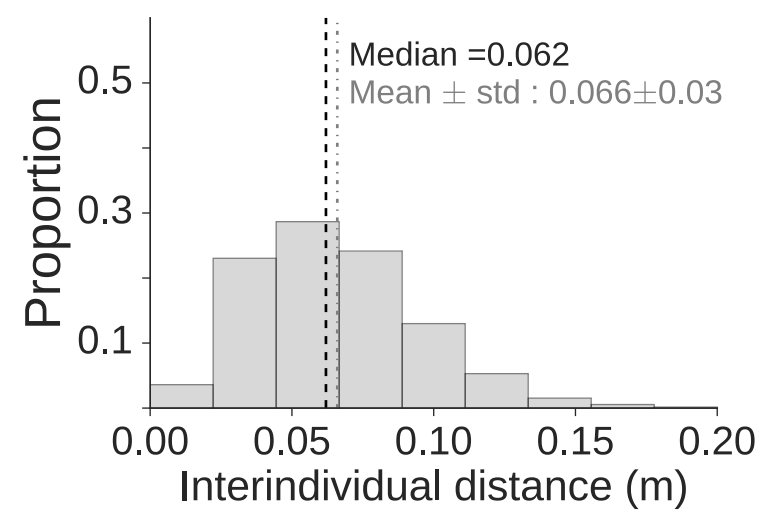

C4

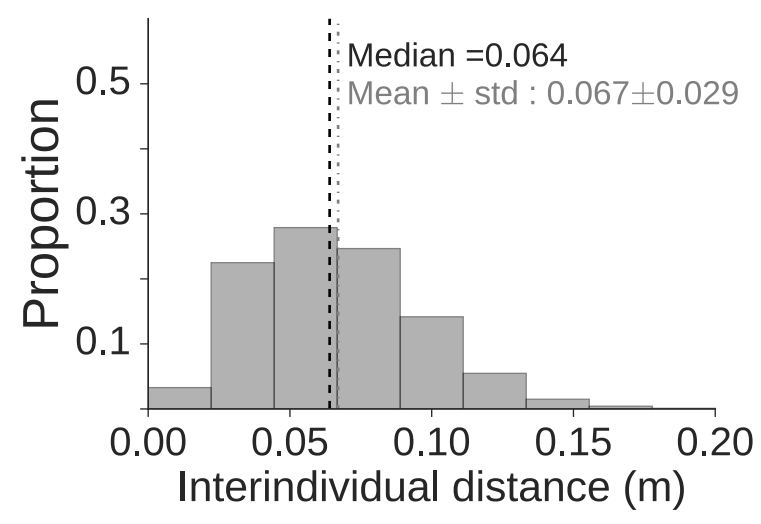

C1

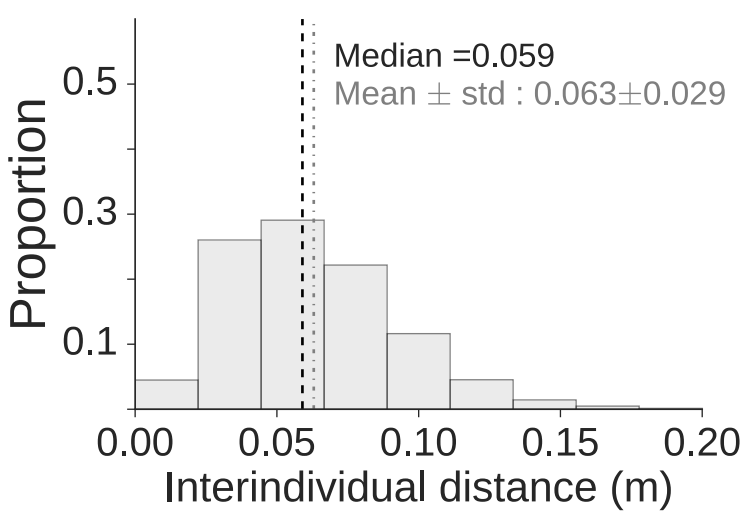

C3

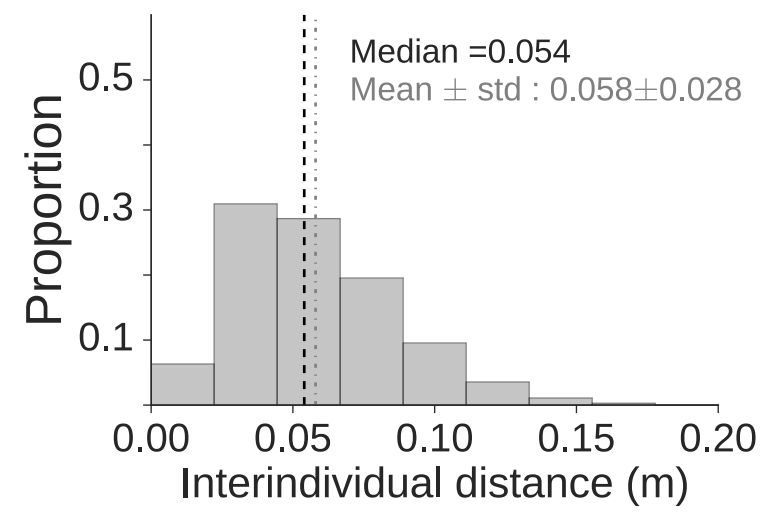

Fig. 6: Mean inter-individual distances between each pair of agents (fish and robot) in all sub-groups. Results are obtained in 30 minutes experiments using groups of wild-type zebrafish moving in the set-up described in Fig. 2A. Each experiment is reiterated ten times. All distributions of inter-individual distances differ significantly (Two-sample Kolmogorov-Smirnov test, with p-values < 0.05; cf Sec. II-D). 
C0

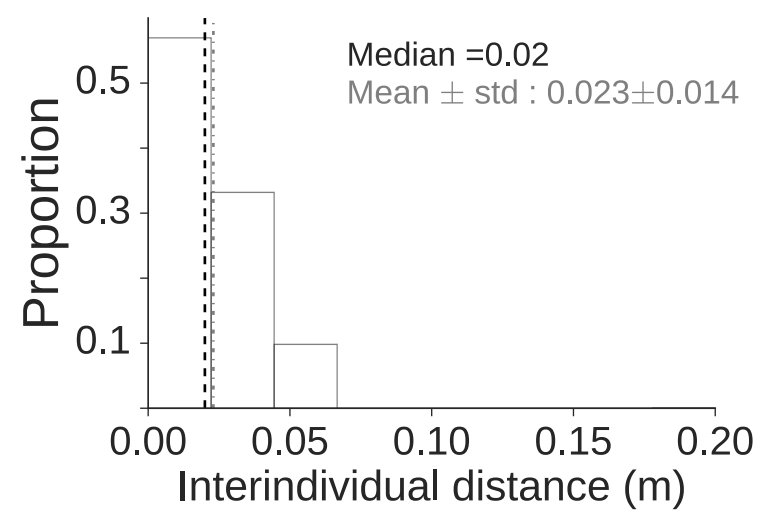

C2

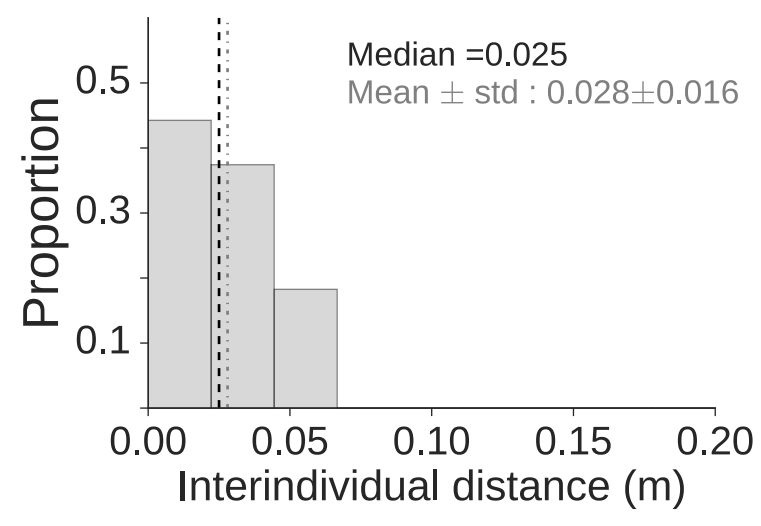

C4

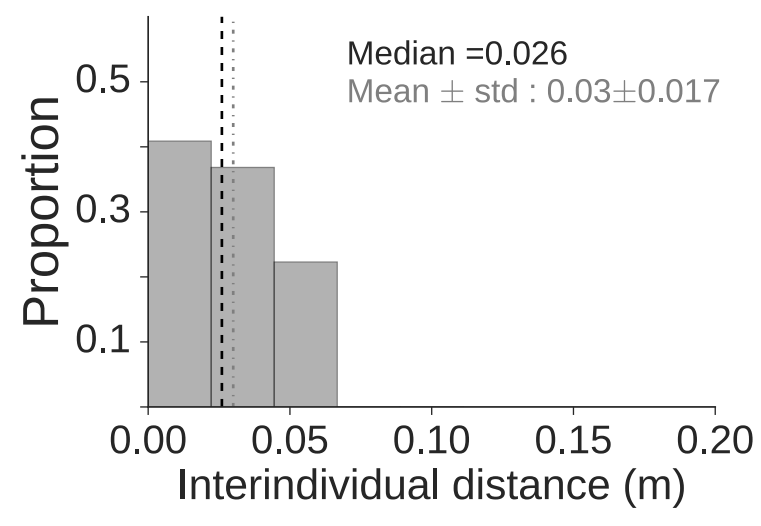

C1

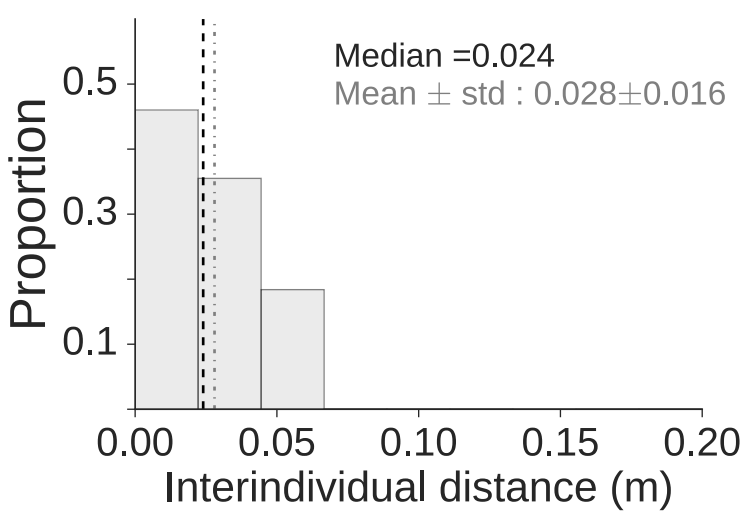

C3

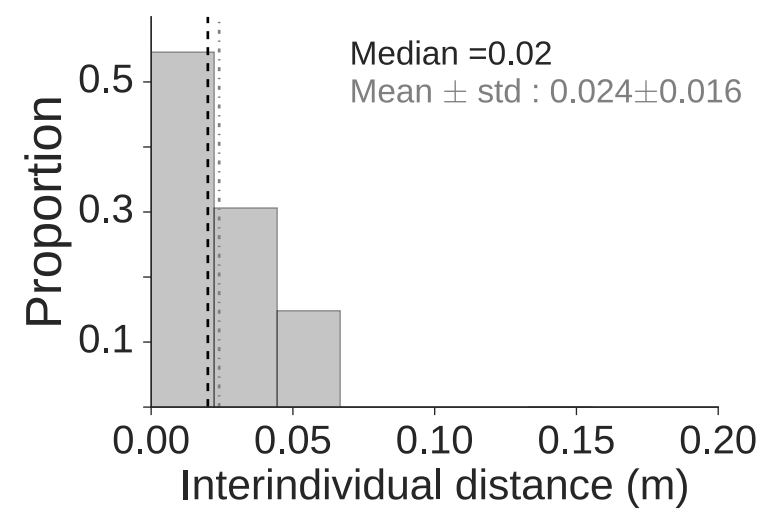

Fig. 7: Mean inter-individual distances between each pair of agents (fish and robot) in sub-groups of only two individuals (pairs). Results are obtained in 30 minutes experiments using groups of wild-type zebrafish moving in the set-up described in Fig. 2A. Each experiment is reiterated ten times. All distributions of inter-individual distances differ significantly (Two-sample Kolmogorov-Smirnov test, with p-values < 0.05; cf Sec. II-D). 
CO

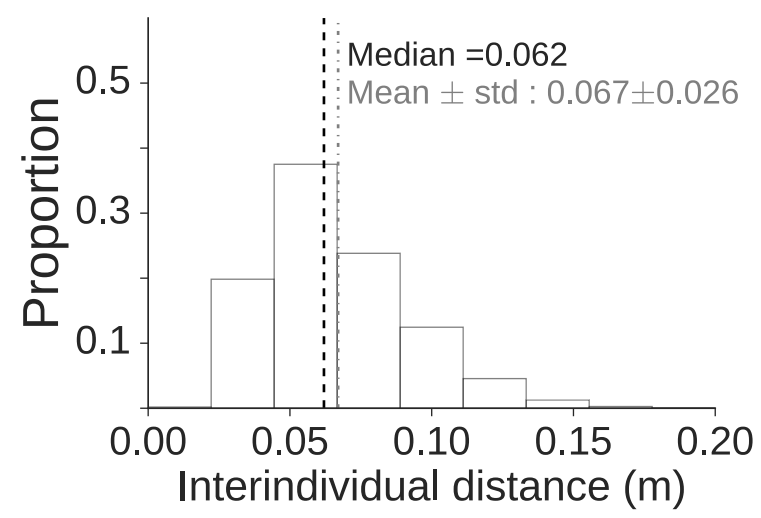

C2

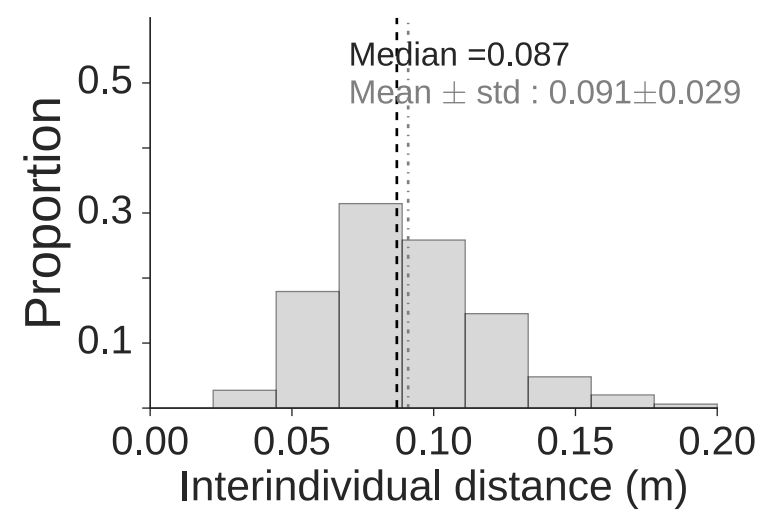

C4

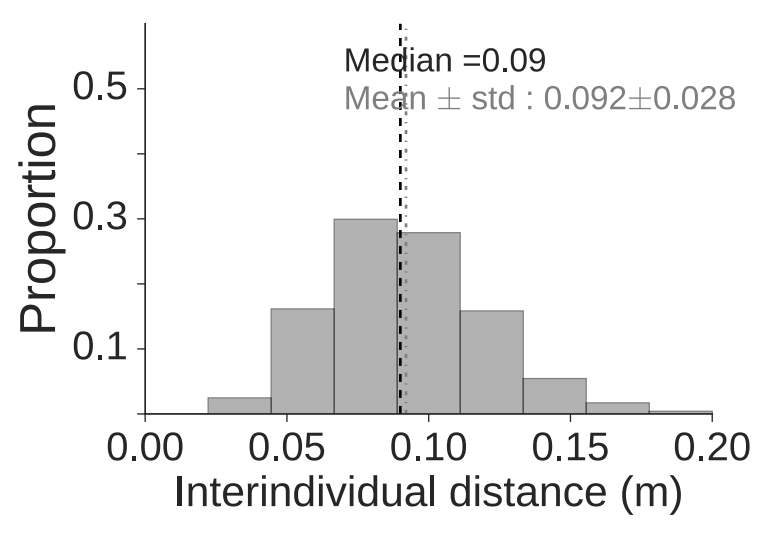

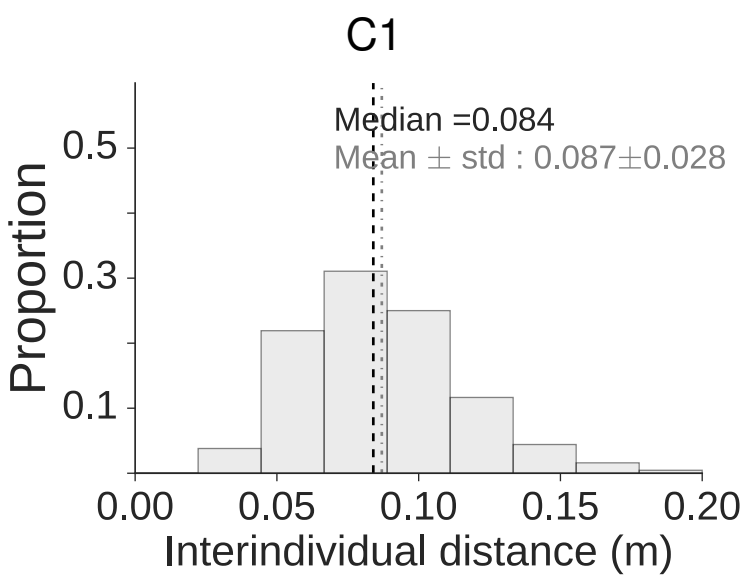

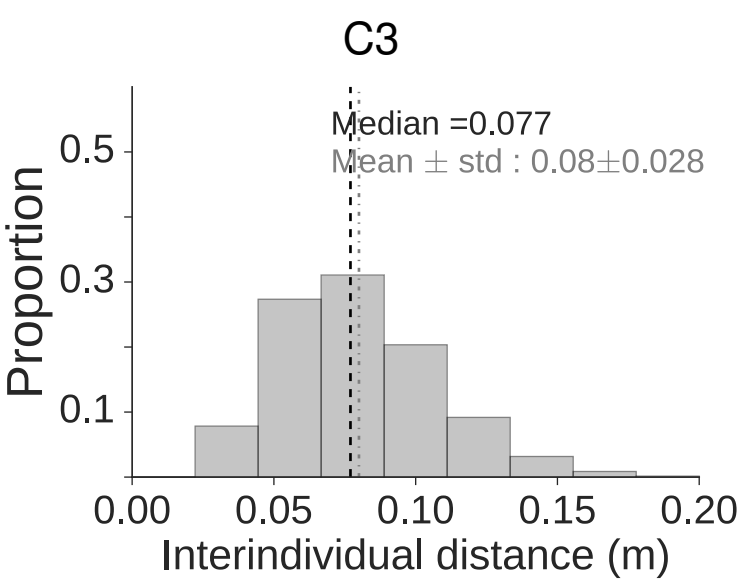

Fig. 8: Mean inter-individual distances between each pair of agents (fish and robot) in sub-groups of five individuals (entire population). Results are obtained in 30 minutes experiments using groups of wild-type zebrafish moving in the set-up described in Fig. 2A. Each experiment is reiterated ten times. All distributions of inter-individual distances differ significantly (Two-sample Kolmogorov-Smirnov test, with p-values $<0.05$; cf Sec. II-D). 
different behaviour depending on sub-group size and composition. While studies in the literature established the existence of these sub-group dynamics in fish [31], no method was described to identify them. We present an algorithm to identify sub-groups of fish based on their spatial proximity. The results of this sub-group analysis show that fish exhibit different behaviours depending on the size of the sub-group they are part of, and of their position in the arena. In particular, fish behave differently when alone compared to when in a group. We showed that the inter-individual distances of fish in a sub-groups is dependant of the size of the sub-group. When they are in the corridor, they tend to pass through quickly from one room to the other.

The social integration of the robot into the groups of fish could still be improved by refining the behavioural model. The model could be further calibrated to take into account more aspects of the fish collective behaviour in this complex environment. The robot behaviour could be closer to the fish behaviour that depends on the size of its sub-group and to its spatial position in the set-up. Our model does not yet take into account explicitly the attraction of the agents towards the walls, like the model in [40]. This was mainly motivated by current technical difficulties as this would greatly increase the number of collisions between the robot and the walls. This problem will be addressed in a subsequent study. Robots can still be seen to be following walls in the colored trajectory presented in Fig. 4 because they are attracted to fish, which, in turn, tend to follow walls.

Future works will focus on automating the calibration of model parameters, by optimising them using evolutionary algorithms. Additionally, the social integration can be further quantified by adding other kind of analysis, and by using more behavioural features in the computation of the social integration indexes.

This study is a first step towards more complex biohybrid groups: instead of just focusing on integration, the robots could be used to control or to modulate the collective behaviour of the mixed group of fish and robots. Indeed taking advantage of specific social behaviours the robots could modulate the whole mixed-groups [10]. The number of robots necessary to have a good control of the mixed groups can also be optimised for example by evolutionary computation [42], [43]. This type of control would be based on natural animal behaviours thus reducing stress and not using coercion to get the target results [4], [10]. Indeed, in zebrafish groups all the fish can be leaders and induce groups transitions from one place to the other. For example, we have shown that the number of initiation is linearly proportional to the number of attempts performed [32]. This allows biomimetic robots to make use of the fish behavioural features to be capable of inducing collective departures and to modulate the spatial distribution of the groups. Such biohybrid social systems would allow us to modulate and to control group living animals.

\section{ACKNOWLEDGMENTS}

This work has been funded by EU-ICT project 'ASSISIbf', no 601074.

\section{REFERENCES}

[1] GL Patricelli, JC Uy, G Walsh, and G Borgia. Sexual selection: male displays adjusted to female's response. Nature, 415(6869):279-280, 2002.

[2] J Knight. Animal behaviour: when robots go wild. Nature, 434(7036):954-955, 2005.

[3] R Vaughan, N Sumpter, J Henderson, A Frost, and S Cameron. Experiments in automatic flock control. Robotics and Autonomous Systems, 31(1):109-117, 2000.

[4] F Mondada, J Halloy, A Martinoli, N Correll, A Gribovskiy, G Sempo, R Siegwart, and JL Deneubourg. A general methodology for the control of mixed natural-artificial societies. In S. Kernbach, editor, Handbook of collective robotics: fundamentals and challenges, chapter 15, pages 547-585. Pan Stanford, 2013.

[5] N Correll, M Schwager, and D Rus. Social control of herd animals by integration of artificially controlled congeners. In International Conference on Simulation of Adaptive Behavior, pages 437-446. Springer, 2008.

[6] F Zabala, P Polidoro, A Robie, K Branson, P Perona, and MH Dickinson. A simple strategy for detecting moving objects during locomotion revealed by animal-robot interactions. Current Biology, 22(14):1344-1350, 2012.

[7] JJ Faria, JRG Dyer, RO Clément, ID Couzin, N Holt, AJW Ward, D Waters, and J Krause. A novel method for investigating the collective behaviour of fish: introducing 'robofish'. Behavioral Ecology and Sociobiology, 64(8):1211-1218, 2010.

[8] Niko Tinbergen. The study of instinct. Clarendon Press/Oxford University Press, 1951.

[9] Daniel Strömbom, Richard P Mann, Alan M Wilson, Stephen Hailes, A Jennifer Morton, David JT Sumpter, and Andrew J King. Solving the shepherding problem: heuristics for herding autonomous, interacting agents. Journal of The Royal Society Interface, 11(100):20140719, 2014.

[10] J Halloy, G Sempo, G Caprari, C Rivault, M Asadpour, F Tâche, I Said, V Durier, S Canonge, and JM Amé. Social integration of robots into groups of cockroaches to control self-organized choices. Science, 318(5853):1155-1158, 2007. 
[11] E Donati, M Worm, S Mintchev, M van der Wiel, G Benelli, G von der Emde, and C Stefanini. Investigation of collective behaviour and electrocommunication in the weakly electric fish, mormyrus rume, through a biomimetic robotic dummy fish. Bioinspiration \& Biomimetics, 11(6):066009, 2016.

[12] DT Swain, ID Couzin, and NE Leonard. Real-time feedback-controlled robotic fish for behavioral experiments with fish schools. Proceedings of the IEEE, 100(1):150-163, 2012.

[13] T Landgraf, H Nguyen, J Schröer, A Szengel, RJG Clément, D Bierbach, and J Krause. Blending in with the shoal: robotic fish swarms for investigating strategies of group formation in guppies. In Biomimetic and Biohybrid Systems, pages 178-189. Springer, 2014.

[14] T Landgraf, D Bierbach, H Nguyen, N Muggelberg, P Romanczuk, and J Krause. Robofish: increased acceptance of interactive robotic fish with realistic eyes and natural motion patterns by live trinidadian guppies. Bioinspiration \& biomimetics, 11(1):015001, 2016.

[15] P Phamduy, G Polverino, RC Fuller, and M Porfiri. Fish and robot dancing together: bluefin killifish females respond differently to the courtship of a robot with varying color morphs. Bioinspiration \& biomimetics, 9(3):036021, 2014.

[16] G Polverino, P Phamduy, and M Porfiri. Fish and robots swimming together in a water tunnel: robot color and tail-beat frequency influence fish behavior. PloS one, 8(10):e77589, 2013.

[17] G Polverino and M Porfiri. Zebrafish (danio rerio) behavioural response to bioinspired robotic fish and mosquitofish (gambusia affinis). Bioinspiration \& biomimetics, 8(4):044001, 2013.

[18] N Abaid, T Bartolini, S Macrì, and M Porfiri. Zebrafish responds differentially to a robotic fish of varying aspect ratio, tail beat frequency, noise, and color. Behavioural brain research, 233(2):545-553, 2012.

[19] S Butail, F Ladu, D Spinello, and M Porfiri. Information flow in animal-robot interactions. Entropy, 16(3):1315-1330, 2014.

[20] F Ladu, T Bartolini, SG Panitz, F Chiarotti, S Butail, S Macrì, and M Porfiri. Live predators, robots, and computer-animated images elicit differential avoidance responses in zebrafish. Zebrafish, 2015.

[21] F Ladu, V Mwaffo, J Li, S Macrì, and M Porfiri. Acute caffeine administration affects zebrafish response to a robotic stimulus. Behavioural brain research, 289:48-54, 2015.

[22] G Polverino, N Abaid, V Kopman, S Macrì, and M Porfiri. Zebrafish response to robotic fish: preference experiments on isolated individuals and small shoals. Bioinspiration \& biomimetics, 7(3):036019, 2012.

[23] C Spinello, S Macrì, and M Porfiri. Acute ethanol administration affects zebrafish preference for a biologically inspired robot. Alcohol, 47(5):391-398, 2013.

[24] G Polverino and M Porfiri. Mosquitofish (gambusia affinis) responds differentially to a robotic fish of varying swimming depth and aspect ratio. Behavioural brain research, 250:133-138, 2013.

[25] T Ruberto, V Mwaffo, S Singh, D Neri, and M Porfiri. Zebrafish response to a robotic replica in three dimensions. Royal Society Open Science, 3(10):160505, 2016.

[26] T Bartolini, V Mwaffo, A Showler, S Macrì, S Butail, and M Porfiri. Zebrafish response to 3d printed shoals of conspecifics: the effect of body size. Bioinspiration \& biomimetics, 11(2):026003, 2016.

[27] M Kruusmaa, G Rieucau, JCC Montoya, R Markna, and NO Handegard. Collective responses of a large mackerel school depend on the size and speed of a robotic fish but not on tail motion. Bioinspiration \& Biomimetics, 11(5):056020, 2016.

[28] N Abaid, S Marras, C Fitzgibbons, and M Porfiri. Modulation of risk-taking behaviour in golden shiners (notemigonus crysoleucas) using robotic fish. Behavioural processes, 100:9-12, 2013.

[29] S Butail, T Bartolini, and M Porfiri. Collective response of zebrafish shoals to a free-swimming robotic fish. PLoS One, 8(10):e76123, 2013.

[30] S Butail, G Polverino, P Phamduy, F Del Sette, and M Porfiri. Influence of robotic shoal size, configuration, and activity on zebrafish behavior in a free-swimming environment. Behavioural brain research, 275:269-280, 2014.

[31] A Séguret, B Collignon, L Cazenille, Y Chemtob, and J Halloy. Loose social organisation of ab strain zebrafish groups in a two-patch environment. arXiv preprint arXiv:1701.02572, 2017.

[32] B Collignon, A Séguret, Y Chemtob, L Cazenille, and J Halloy. Collective departures in zebrafish: profiling the initiators. arXiv preprint arXiv:1701.03611, 2017.

[33] F Bonnet, L Cazenille, A Gribovskiy, J Halloy, and F Mondada. Multi-robots control and tracking framework for bio-hybrid systems with closed-loop interaction. In Robotics and Automation (ICRA), 2017 IEEE International Conference on. IEEE, 2017.

[34] F Bonnet, P Rétornaz, J Halloy, A Gribovskiy, and F Mondada. Development of a mobile robot to study the collective behavior of zebrafish. In 2012 4th IEEE RAS \& EMBS International Conference on Biomedical Robotics and Biomechatronics (BioRob), pages 437-442. Ieee, 2012.

[35] F Bonnet, S Binder, ME de Oliveria, J Halloy, and F Mondada. A miniature mobile robot developed to be socially integrated with species of small fish. In Robotics and Biomimetics (ROBIO), 2014 IEEE International Conference on, pages 747-752. IEEE, 2014.

[36] F Bonnet, L Cazenille, A Seguret, B Collignon, J Halloy, and F Mondada. Design of a modular robotic system that mimics small fish locomotion and body movements for ethological studies. International Journal of Advanced Robotic Systems, 14(3):1729881417706628, 2017.

[37] F. Bonnet, N. Crot, D. Burnier, and F. Mondada. Design methods for miniature underwater soft robots. In 2016 th IEEE International Conference on Biomedical Robotics and Biomechatronics (BioRob), pages 1365-1370, June 2016.

[38] M Deza and E Deza. Dictionary of distances. Elsevier, 2006.

[39] Adam Zienkiewicz, David AW Barton, Maurizio Porfiri, and Mario Di Bernardo. Data-driven stochastic modelling of zebrafish locomotion. Journal of mathematical biology, 71(5):1081-1105, 2015. 
[40] B Collignon, A Séguret, and J Halloy. A stochastic vision-based model inspired by zebrafish collective behaviour in heterogeneous environments. Royal Society Open Science, 3(1):150473, 2016.

[41] V Braitenberg. Vehicles: Experiments in synthetic psychology. MIT press, 1986.

[42] L Cazenille, N Bredeche, and J Halloy. Multi-objective optimization of multi-level models for controlling animal collective behavior with robots. In Conference on Biomimetic and Biohybrid Systems, pages 379-390. Springer, 2015.

[43] L Cazenille, N Bredeche, and J Halloy. Automated optimisation of multi-level models of collective behaviour in a mixed society of animals and robots. arXiv preprint arXiv:1602.05830, 2016. 